\title{
Additive Property and the Physical Reducibility of the Mind
}

\author{
Kwangho Hyun \\ School of Medicine, The Catholic University of Korea, Seoul, South Korea \\ Email: kwangho.hyun@gmail.com
}

Received 28 January 2014; revised 28 February 2014; accepted 7 March 2014

Copyright (C) 2014 by author and Scientific Research Publishing Inc.

This work is licensed under the Creative Commons Attribution International License (CC BY). http://creativecommons.org/licenses/by/4.0/

c) (i) Open Access

\begin{abstract}
It is controversial whether the mind is physically reducible or not. If the mind is physically reducible, it must share properties with the physical world. To find out how the mind can be characterized, an "additive property" is used to describe the mind. The additive property is shown where many causes result in one effect. The additive property is shown in the biological domain when the senses process received input. It is also shown in the intellectual activities of daily life. In the physical world, there is no cause without an effect. The additive property of events is not satisfied in physical domain. If the mind is physically reducible, the additive property of the mind should not be satisfied. Because the additive property is shown in the mind, the mind cannot be physically reduced in terms of relationships of cause and effects.
\end{abstract}

\section{Keywords}

Additive Property; Reducibility; Causality; Neuron; Mind Body

\section{Introduction}

Whether the mind is physically reducible or not is a topic that is still controversial. Supervenience provides us a powerful tool to analyze how the mind is related to the physical domain. It is the biological nature that comprises the mental activities. The additive property of an event is another characteristic that is often presented in the biological domain. Especially in neurons, the existence of an additive property in neural activities is definite. To differentiate the physical domain and the biological domain with the mental domain, an additive property is discussed to consider whether the mind is physically reducible.

\section{Additive Property of an Event}

In an event, an additive property may or may not hold. In this paper, I will express an event with an implication. 
For example, $\mathrm{x}+\mathrm{y} \rightarrow \mathrm{z}$ will express an event. The left side of the implication denotes the cause. Here, both $\mathrm{x}$ and $y$ designate two different causes. The right side of the implication denotes the effect. Here, there is only one effect $z$. The operation + expresses the relation of causes to result in an output effect. Two or more elements on the left side of the implication will result in a single element on the right side of the implication. In other words, two or more causes will result in one effect. This will be described as the additive property.

The main idea of the additive property is to admit a cause without an effect. In case of $x+y \rightarrow z$, neither $x$ nor y should have effect. Only one effect out of many causes will be plausible. It is difficult to find such cases in ordinary physics. In neurons, this occurs frequently when making action potentials.

\section{Additive Property in Physics}

Before discussing the additive property in physics, events in physics must be explained. In the physical domain, all events are preserved. No physical event has a cause outside the physical domain (Kim, 1993). From this point of view, no event appears and no event disappears in the physical domain. In other words, there are no missing matches between causes and effects in events. It has been mentioned that, in fields of science, there is no cause without an effect (Thilly, 1901; Goldenweiser, 1938). Physics is a representative field of science. A cause without an effect is not generally accepted.

The additive property does not hold in the physical domain. The implication $\mathrm{x}+\mathrm{y} \rightarrow \mathrm{z}$ denotes the additive property. The left side of the implication denotes that two causes, $\mathrm{x}$ and $\mathrm{y}$, are related with the additive property to effect in effect $\mathrm{z}$ on the right side of the implication. If no physical cause and effect relationship disappears, the number of causes and effects must be preserved. In this case, the two causes on the left led to one effect on the right. The numbers of causes and effects on the two side of implication do not match. Thus, the additive property does not hold in the physical domain.

\section{Additive Property in Neurons}

Contrary to physics, the additive property holds in the biological world. In neurons, both excitatory postsynaptic potential (EPSP) and inhibitory postsynaptic potential (IPSP) exists. In EPSP, amino acid glutamine often functions as a key to open ion channels in postsynaptic cell membranes. Positive charged extracellular ions penetrate into postsynaptic cell membranes to form an action potential. Because there are many axon terminals from many different neurons that reach dendrites in a single neuron, the addition of several EPSPs will result in a single action potential in the neuron shown in Figure 1. More specifically, if numerical addition of each small EPSP voltage exceeds the threshold at specific time interval, one single action potential will fire.

As seen in Figure 2, many different neurons that have axon 1, axon 2, and axon 3 will make different EPSP to form a single action potential in axon 4 . In this case, we can see that $x+y+z \rightarrow w$. Here, $x$ is the EPSP in axon $1, \mathrm{y}$ is the EPSP in axon 2, $\mathrm{z}$ is the EPSP in axon 3, and $\mathrm{w}$ is the action potential in axon 4. If numerical addition of EPSP voltage $x, y, z$ exceeds a certain threshold, action potential $w$ will fire. As Figure 2 shows, the

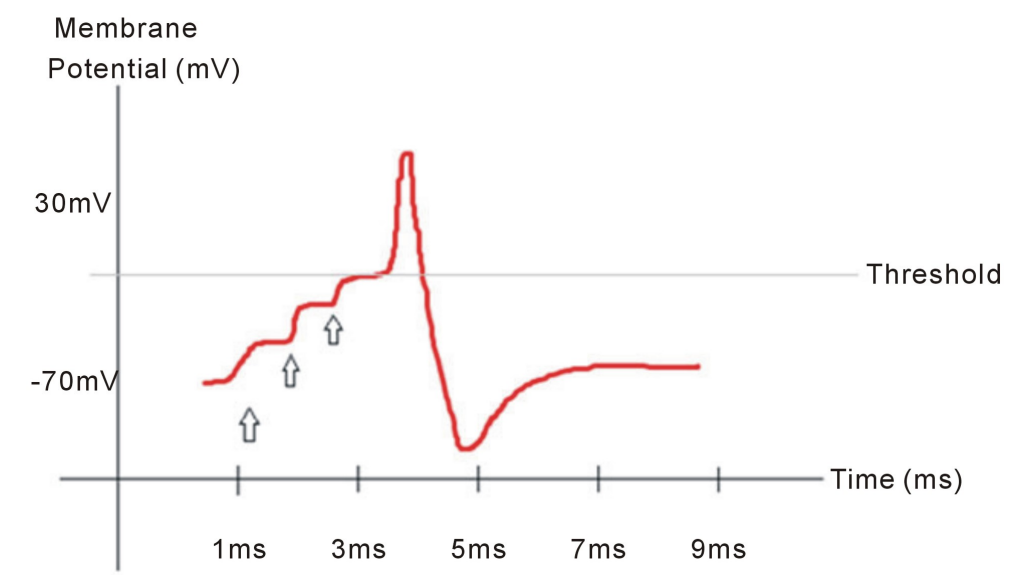

Figure 1. A single action potential firing event: summation of excitatory postsynaptic potentials (EPSP) to form an action potential. 


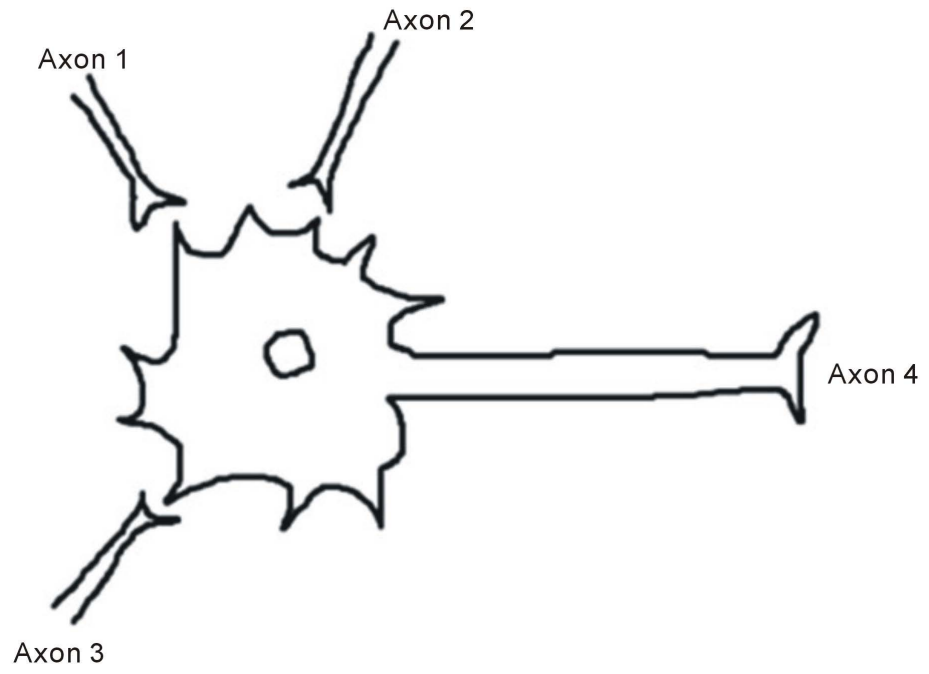

Figure 2. Summation of signals from several axon terminals in a neuron.

additive property of an event holds in neurons.

A cause without an effect can be found here. If any EPSP that does not reach the threshold exists, no action potential will fire. Even though EPSP is strictly classified as the cause of action potential firing, no firing will result if the sum of EPSP does not exceed the threshold. This is different from the physical domain, where there is no cause without an effect. In the case of neuron action potential firing, it is natural to have a cause without an effect. In summary, the additive property of an event is satisfied in the biological domain. This supports the existence of a cause without an effect.

\section{Supervenience}

Supervenience is a notion that explains mental property in relationship with physical properties. It originates from moral philosophy and is well shown in a passage from G. E. Moore (Kim, 1984). It is often claimed that mental property M supervenes upon physical property P. More specifically, it can be stated as the claim that "there cannot be an A difference without B difference." This briefly explains the property of supervenience. For example, the color and shape of a picture supervenes upon molecular and physical properties in real world on earth. The photons from external light bounce off an atom to show colors. The nature of the picture is based upon the molecular arrangements of pigments. As a result, the picture supervenes upon physical properties.

There are two basic types of supervenience, namely, weak supervenience, and strong supervenience. Weak supervenience gives us a tool to differentiate targets. For example, if there are given individuals $\mathrm{x}$ and $\mathrm{y}$, weak supervenience tells us how they are related by given properties or not. On the other hand, strong supervenience gives us a tool to further generalize how any individual can be related. For example, if there are some properties that are shown, any individuals with those properties may be related (Kim, 1984).

More technically, we can further discuss weak supervenience and strong supervenience. For example, A weakly supervenes on $\mathrm{B}$ if and only if $\mathrm{x}$ and $\mathrm{y}$ share all properties in $\mathrm{B}$, then $\mathrm{x}$ and $\mathrm{y}$ share all properties in $\mathrm{A}$. Weak supervenience tells us the indifferentiability between given individuals. While weak supervenience gives us indifferentiability between given individuals, strong supervenience gives a tool to further generalize relationships between any individuals. Thus, A strongly supervenes on B if the following conditions are satisfied. A strongly supervenes on $B$, if for each element $x$ in $A$ that has property $G$ in $A$, and if $x$ has property $F$ in $B, A$ strongly supervenes on B if any element $y$ in B that shows property $F$ necessarily has property $G$ in $A$.

The biological world can be modeled as a biological domain. The physical world can be modeled as a physical domain. Likewise, the mental world can be modeled as a mental domain. These logical descriptions, such as domains, are used to compare how those domains supervene on each other. If they do not supervene on each other, one may not be reduced to the other. To have the basic requirements for reducibility, supervenience is a necessary condition. If one thing does not strongly supervene on another, this implies that two different domains 
do not share the same properties. If two different domains do not share the same properties, and if these properties are basic requirements for the establishment of these domains, they are not reducible to each other. To generalize how any elements in these domains supervene each other, strong supervenience is used for the discussion.

\section{Strong Supervenience and the Additive Property of the Mind}

From observations, the additive property of mind is satisfied in both the mental and biological domains. Let's assume that in biological domain $\mathrm{A}$, there is a tasting event $\mathrm{x}$ that shows additive property $\mathrm{G}$. For example, bitter and sweet EPSP input signals from sensory receptors both pass a neuron to be combined into a single output action potential signal that informs someone that he or she just tasted cough syrup. This represents the additive property of the biological domain. Let's assume that in mental domain $\mathrm{B}$, a tasting event $\mathrm{x}$ has both sweet taste and bitter taste to form a single sense of cough syrup taste. Both sweet taste and bitter taste are summed up into a sense of cough syrup. This is what can be observed. Because we recognize both sweet sense and bitter sense as a single sense of cough syrup in the mental domain, the additive property is satisfied in both the biological domain and the mental domain.

Let's assume that the mind is biologically reducible. This requires the mental domain and biological domain to have a relationship called supervenience. By using the definition of strong supervenience, we can find out whether the mental domain supervenes on the biological domain. In biological domain $\mathrm{A}$, tasting event $\mathrm{x}$ has additive property $\mathrm{G}$. In mental domain $\mathrm{B}$, the same tasting event $\mathrm{x}$ has the additive property $\mathrm{F}$. Tasting event $\mathrm{x}$ in mental domain $\mathrm{B}$ shows additive property $\mathrm{F}$ which necessarily presents additive property $\mathrm{G}$ in biological domain A. Mental domain B strongly supervenes on biological domain A. Thus, the mind satisfies the basic requirements for biologically reducibility.

Let's assume that the mind is physically reducible. By using the definition of strong supervenience, we can find out whether the mental domain supervenes on the physical domain. In physical domain A, tasting event $\mathrm{x}$ does not have additive property $\mathrm{G}$. In mental domain $\mathrm{B}$, the same tasting event $\mathrm{x}$ has the additive property $\mathrm{F}$. Tasting event $\mathrm{x}$ in mental domain $\mathrm{B}$ shows additive property $\mathrm{F}$ which does not necessarily present additive property $\mathrm{G}$ in physical domain $\mathrm{A}$. The mental domain does not strongly supervene on physical domain $\mathrm{A}$. This also means that the mental domain does not weakly supervene on the physical domain. This is not supervenience. Because the mental domain and the physical domain do not satisfy the basic requirements for supervenience, the mind is not physically reducible.

Furthermore, let's assume that the biological domain is physically reducible. By using the definition of strong supervenience, we can find whether the biological domain supervenes on the physical domain. Supervenience is necessary for the reducibility of the biological domain to the physical domain. In physical domain A, the action potential firing event $x$ does not have additive property $G$. In biological domain $B$, the same action potential firing event $\mathrm{x}$ has the additive property $\mathrm{F}$. Action potential firing event $\mathrm{x}$ in physical domain A does not show additive property F. Action potential firing event $\mathrm{x}$ in biological domain $\mathrm{B}$ shows additive property $\mathrm{G}$. The biological domain does not strongly supervene on physical domain A. The biological domain does not supervene on the physical domain. Because the basic requirements for reducibility are rejected, the biological domain is not physically reducible.

Davidson claimed that mental events supervene on physical characteristics (Davidson, 1970). From this context, he clearly meant that physical characteristics are the molecular actions of neurons and action potentials rather than event theories and strings with elementary particles. Action potentials and biological mechanisms are truly physically dependent. Positive ions and molecular ion channels comprise the actions of neuronal activities. The single firing of an action potential combines many separate events into a single output event. At this point, the biological activity does not satisfy the causal closure shown in the physical domain. The mental domain supervenes on the biological domain. It should be the point what Davidson mentioned from the context. The biological domain does not supervene on the physical domain; thus, the mind is not physically reducible.

By using this logic, we can further discuss belief, which is one of the characteristics of the mind. Let's assume that person $\mathrm{P}$ has a belief that ginger is always sweet. In any case, he will admit that ginger is always tasty. Let's assume that the taste of ginger is x. Let's also assume that the taste of a cookie is $\mathrm{y}$. If that person had tasted both ginger and a cookie, he will believe that ginger is sweet whenever he eats any kind of cookie with ginger. Let's assume that there is an event such as tasting both ginger and a cookie. In this event, ginger is cause $\mathrm{x}$ and a 
cookie is cause $y$. Because ginger is always tasty, $x+y \rightarrow x$ with any $y$. If we set $y$ to 0 , the implication that describes this event successfully describes the additive property. Thus, belief can be classified as a phenomenon that shows a kind of additive property. A cause without an effect exists here. Because showing additive property is not physically reducible, belief may not be physically reducible.

\section{Acknowledgements}

I would like to thank Professor Jaegwon Kim for giving me the first impression to modern philosophy. Professor Kim has given me kind considerations for years. I would also like to thank Professor Woosuk Park for helping me develop ideas in undergraduate courses. Professor Park also helped me in the revision process of this paper. Main insights of additive property were from investigating In-yeon (因縁, pațicca-samuppāda), which is one of the main ideas in Buddhism.

\section{References}

Davidson, D. (1970). Mental Events. In L. Foster, \& J. W. Swanson (Eds.), Experience and Theory (pp. 79-101), Amherst: University of Massachusetts Press.

Goldenweiser, A. (1938). The Concept of Causality in the Physical and Social Sciences, American Sociological Review, 3 , 624-636. http://dx.doi.org/10.2307/2084682

Kim, J. (1984). Concepts of Supervenience. Philosophy and Phenomenological Research, 45, 153-176. http://dx.doi.org/10.2307/2107423

Kim, J. (1987). “Strong” and “Global” Supervenience Revisited. Philosophy and Phenomenological Research, 47, 315-328. http://dx.doi.org/10.2307/2107631

Kim, J. (1993). Supervenience and Mind: Selected Philosophical Essays (p. 280). Cambridge: Cambridge University Press. http://dx.doi.org/10.1017/CBO9780511625220

Thilly, F. (1901). The Theory of Interaction. Philosophical Review, 10, 124-138. http://dx.doi.org/10.2307/2176507 